\title{
5 SITUAÇÕES INDUTORAS DE STRESS E BURNOUT EM ESTUDANTES DE ENFERMAGEM NOS ENSINOS CLÍNICOS
}

\author{
| Conceição Martins'; Sofia Campos²; João Duarte ; Rosa Martins'; Teresa Moreira ; Cláudia Chaves ${ }^{6}$ |
}

\section{RESUMO}

CONTEXTO: A exposição recorrente a situações de Stress pode levar a Burnout, síndrome psicológica em resposta a fatores de Stress crónicos no trabalho. O ensino de Enfermagem desperta interesse relativamente ao Stress e Burnout dos futuros enfermeiros, pois considera-se que os estudantes vivenciam situações, que sendo stressantes para os enfermeiros, também o podem ser para eles.

OBJETIVO(S): Identificar situações indutoras de Stress e Burnout em estudantes de enfermagem nos ensinos clínicos; Analisar a influência das variáveis sociodemográficas e académicas no Stress e Burnout.

METODOLOGIA: Estudo quantitativo e descritivo e correlacional. Amostra de 236 estudantes em ensino clínico, recolha de dados com questionário sociodemográfico e caracterização académica, Escala KEZKAK e Inventário de Burnout de Maslach.

RESULTADOS: A amostra demonstra índices de saúde baixos; Os enfermeiros estão vulneráveis ao stress; O stress produzido pelas circunstâncias organizacionais, a totalidade da amostra revela elevados índices de stress e o seu bem-estar biopsicossocial, manifestamente afetado. CONCLUSÕES: Amostra maioritariamente feminina (78.4\%), idade média 21.17 anos ( \pm 2.487 Anos); prevalências de estudantes a frequentarem o $3^{\circ}$ e $4^{\circ}$ ano (42.8\% vs. $\left.43.2 \%\right)$. Predomínio de Stress moderado (47.0\%). Situações que causam Stress aos estudantes: "Não controlar a relação com o doente/utente" 49.36 ( \pm 16.458$)$, "Falta de competência" (M=48.55; \pm 21.043$)$, "Relação com supervisor e colegas" ( $M=44,95)$, "Sobrecarga" (M=40,85), "Impotência e incerteza" $(\mathrm{M}=44.43)$. Prevalência de baixo Burnout com percentuais mais elevados em todas as variáveis sociodemográficas e académicas. As variáveis sociodemográficas e académicas com significado estatístico no Burnout foram o sexo, a idade e a coabitação em tempo de aulas.

PALAVRAS-CHAVE: Stresse Psicológico; Esgotamento Profissional; Educação em Enfermagem; Estudantes de Enfermagem

\section{RESUMEN}

\section{"Situaciones inductores de estrés y el desgaste en los estudiantes de enfermería en enseñanzas clínicos"}

ANTECEDENTES: La exposición repetida a situaciones de estrés puede conducir al desgaste, síndrome psicológico en respuesta a factores de estrés crónico en el trabajo. La formación de enfermería despierta el interés en relación con el estrés y agotamiento de las enfermeras futuro ya que se considera que los estudiantes experimentan situaciones que son estresantes para las enfermeras, también puede ser para ellos.

OBJETIVO (S): Identificar situaciones inductoras de estrés y agotamiento de los estudiantes de enfermería en la enseñanza clínica; Para analizar la influencia de las variables sociodemográficas y académicas sobre estrés y agotamiento.

MÉTODOS: Estudio cuantitativo y descriptivo y correlacional. Muestra de 236 estudiantes en la formación clínica, la recopilación de datos con el cuestionario sociodemográfico y caracterización académica, Escala KEZKAK y Maslach Burnout Inventory.

RESULTADOS: La muestra presenta bajos indicadores de salud; Las enfermeras son vulnerables al estrés; El estrés producido por circunstancias de la organización, toda la muestra revela altos niveles de estrés y su bienestar biopsicosocial claramente afectado.

CONCLUSIONES: Muestra en su mayoría mujeres $(78,4 \%)$, años de edad promedio 21:17 ( \pm 2.487 Años); prevalencia de estudiantes del $3^{\circ}$ y $4^{\circ}$ año $(42,8 \%$ vs. $43,2 \%)$. Predominio de estrés moderado $(47,0 \%)$. Situaciones que causan estrés a los estudiantes: "No controlar la relación con el paciente / usuario" 49.36 ( \pm 16.458$)$, "falta de competencia" $(\mathrm{M}=48.55 ; \pm 21043)$, "Relación con el supervisor y colegas" $(M=44,95)$, "sobrecarga" $(M=40.85)$, "La impotencia y la incertidumbre" ( $\mathrm{M}=44.43)$. Baja prevalencia de la quemadura con porcentajes más altos en todas las variables sociodemográficas y académicas. Las variables sociodemográficas y académicas con significación estadística en el agotamiento fueron el sexo, la edad y la convivencia en las clases de tiempo.

\section{DESCRIPTORES: El Estrés Psicológico; Agotamiento Profe- sional; Educación en Enfermería; Estudiantes de enfermería}

\begin{abstract}
"Stress and burnout induction situations in nursing students in clinical teaching"

CONTEXT: Recurrent exposure to Stress can lead to Burnout, psychological syndrome in response to chronic Stress factors at work. Nursing education arouses interest in future nurses' Stress and Burnout, as it is considered that students experience situations that are stressful for nurses, so can they be for them.

OBJECTIVE (S): To identify situations inducing Stress and Burnout in nursing students in clinical teaching; To analyze the influence of sociodemographic and academic variables on Stress and Burnout.

METHODOLOGY: Quantitative and descriptive and correlational study. Sample of 236 students in clinical teaching, data collection with sociodemographic questionnaire and academic characterization, KEZKAK Scale and Maslach Burnout Inventory.

RESULTS: The sample shows low health indexes; Nurses are vulnerable to stress; The stress produced by the organizational circumstances, the entire sample shows high stress levels and their biopsychosocial well-being, manifestly affected.

CONCLUSIONS: A predominantly female sample (78.4\%), mean age 21.17 years ( \pm .2 .487 years); Prevalence of students attending the 3 rd and 4 th year ( $42.8 \%$ vs. $43.2 \%)$. Prevalence of moderate stress (47.0\%). Situations that cause Stress to students: "Do not control the relationship with the patient / patient" 49.36 ( \pm 16.458$)$, "Lack of competence" ( $\mathrm{M}=48.55, \pm 21.043)$, "Relationship with supervisor and colleagues" 95$)$, "Overload" $(\mathrm{M}=40.85)$, "Impotence and uncertainty" ( $\mathrm{M}=44.43)$. Prevalence of low Burnout with higher percentages in all sociodemographic and academic variables. The sociodemographic and academic variables with statistical significance in Burnout were gender, age and cohabitation in class time.
\end{abstract}

KEYWORDS: Stress Psychological; Burnout, Professional; Education, Nursing; Students, Nursing

Submetido em 18-12-2016

Aceite em 20-04-2017

1 Doutorada em Ciências da Educação, Professora Coordenadora em Escola Superior de Saúde-Instituto Politécnico de Viseu, Portugal, mcamartinsp@gmail.com 2 Doutorada em Ciências da Educação, Professora Convidada em Escola Superior de Saúde-Instituto Politécnico de Viseu, Portugal, sofiamargaridacampos@gmail.com 3 Doutorado em Ciências da Saúde, Professor Coordenador em Escola Superior de Saúde-Instituto Politécnico de Viseu, Portugal, duarte.johnny@gmail.com

4 Doutorada em Desenvolvimento e Intervenção Psicológica. Professora Coordenadora em Escola Superior de Saúde-Instituto Politécnico de Viseu, rmartins.viseu@gmail.com 5 Mestre em Ciências do Desporto, Professora em Durant Academy Boarding School, St Cuthmans. Midhurst, UK, tmotricidade@gmail.com

6 Doutorada em Ciências da Educação. Professora Adjunta na Escola Superior de Saúde-Instituto Politécnico de Viseu, Portugal, claudiachaves21@gmail.com

Citação: Martins, C., Campos, S., Duarte, J., Martins, R., Moreira, T., \& Chaves, C. (2017). Situações indutoras de stress e Burnout em estudantes de enfermagem nos ensinos clínicos. Revista Portuguesa de Enfermagem de Saúde Mental (Spe. 5), 25-32 


\section{INTRODUÇÃO}

A filosofia subjacente ao cuidar advoga que não basta tratar a pessoa na sua dimensão biológica, mas que a pessoa deve ser vista como um todo, de forma holística, contribuindo esta visão para o seu crescimento e desenvolvimento integral e harmonioso, o que implica que se fomentem estes valores nos ensinos clínicos em Enfermagem. Para que se aprofundasse a compreensão da realidade dos ensinos clínicos à luz de "vidas havidas e vidas a haver" (Ferreira, 2015), o que leva a encará-los como um espaço de confluência entre vários agentes - professores, colegas, orientadores, doentes/familiares e outros profissionais de saúde - o que os transforma num nicho primordial de socialização, porque neles interagem múltiplas pessoas, com todas as suas particularidades. Resultam daqui algumas inquietações que jamais ficarão resolvidas, uma vez que a experiência profissional e pessoal será sempre matizada com singularidades (Ferreira, 2015). Tornase, no entanto, fundamental compreender primeiramente o conceito de stress, dado que se apresenta algo complexo e abrangente. $\mathrm{O}$ stress constitui um termo de origem anglo-saxónica, cuja tradução para a língua portuguesa foi efetivada em 2003, optando nós pela utilização do termo original, pelo facto de nos fazer mais sentido dada a sua internacionalização. De facto, são vários os autores que se dedicam ao estudo do stress, considerando que as suas repercussões no indivíduo são multivariadas, atingindo a sua esfera social, económica, cultural e biológica, sendo que estas são tanto mais acentuadas, quanto mais intensa e prolongada for a vivência de situações desencadeadoras das mesmas (Serra, 2008).

No âmbito destas constatações, emerge a noção do stress provocado pela atividade profissional e as suas repercussões no trabalho, o qual fundamenta a problemática em estudo, sem se querer, contudo, menosprezar as restantes dimensões que o stress abrange. Desde há uns anos que a problemática do stress tem sido estudada e os resultados descrevem que a Enfermagem é uma profissão onde o stress é uma constante. Por outro lado, verifica-se que o contacto com o ensino de Enfermagem desperta interesse relativamente ao stress dos futuros profissionais, pois considera-se que os estudantes em ensino clínico vivenciam situações, que sendo potencialmente stressantes para os enfermeiros, também o podem ser para eles.

\section{METODOLOGIA}

É uma investigação quantitativa do tipo descritivo e correlacional que nos permitirá traçar um perfil sociodemográfico dos estudantes de enfermagem em ensino clínico, determinar quais as variáveis sociodemográficas que interferem no Burnout nos estudantes; analisar a relação entre as variáveis académicas e o Burnout, bem como analisar a relação entre o stress com o Burnout em estudantes de enfermagem em ensino clínico Tendo por base a problemática, delinearam-se as seguintes questões que norteiam esta investigação: i) Qual a prevalência de stress e Burnout nos estudantes de enfermagem em ensino clínico? ii) De que modo as variáveis sociodemográficas interferem no Burnout nos estudantes de enfermagem em ensino clínico? iii) Que variáveis académicas interferem no Burnout nos estudantes de enfermagem em ensino clínico? iv) Qual a relação do stress com o Burnout em estudantes de enfermagem em ensino clínico? Deste modo, delinearam-se os seguintes objetivos, que vão ao encontro das questões de investigação, determinar a prevalência de stress e Burnout nos estudantes de enfermagem em ensino clínico; identificar quais as variáveis sociodemográficas que interferem no Burnout nos estudantes de enfermagem em ensino clínico; analisar a relação entre as variáveis académicas e o Burnout nos estudantes de enfermagem em ensino clínico; analisar a relação entre o stress e o Burnout em estudantes de enfermagem em ensino clínico. Para a realização deste estudo, considerou-se como população os estudantes de enfermagem em ensino clínico da Escola Superior de Saúde de Viseu, donde se extraiu a amostra, que é não probabilística por conveniência. Assim, a amostra ficou constituída por 236 estudantes de enfermagem em ensino clínico.

O protocolo de recolha de dados utilizado foi constituído por um questionário de caracterização sociodemográfica da amostra contém 5 questões, na maioria fechadas (sexo, idade, onde vive habitualmente, com quem vive durante o período letivo, à exceção da questão relativa ao estado civil que é fechada e aberta. Por um questionário de caracterização académica, o mesmo contém duas questões, uma relativa ao ano em que os estudantes estão inscritos (questão fechada) e outra referente ao ensino clínico em que se encontram (questão aberta), ainda, a Escala KEZKAK - Instrumento de medida dos fatores de stress dos estudantes de enfermagem na prática clínica, cujo original de $\mathrm{Zu}$ piria Gorostidi e colaboradores (2003), foi traduzido e adaptado para a língua portuguesa por Barroso et al. (2008). 
A escala KEZKAK original foi construída em Castelhano e Basco e é um importante instrumento de medida de situações identificadas por Zupiria Gorostidi et al. (2003) em relação às quais os estudantes expressam a intensidade do stress experienciado numa escala de resposta tipo Likert de quatro níveis: Nada stressante $(\mathrm{N})=0$; Algo stressante $(\mathrm{A})=1$; Muito stressante $(\mathrm{M})$ $=2$; Muitíssimo stressante $(\mathrm{MT})=3$. A pontuação total permite conhecer como os estudantes consideram as situações em causa e em que medida podem ser stressantes. Assim, valores elevados indicam que os estudantes estão muito preocupados e que as situações são percebidas como stressantes, como também a pontuação total obtida para cada fator permite hierarquizar os fatores particularmente stressantes. $\mathrm{Zu}$ piria Gorostidi et al. (2003) realizaram uma análise fatorial aos 41 itens da escala cujos resultados permitiram agregar, por ordem de maior importância, nove fatores: 1-falta de competência; 2-contacto com o sofrimento; 3-relação com orientadores e colegas; 4-impotência e incerteza; 5-não controlar a relação como o doente/utente; 6-implicação emocional; 7-prejudicar-se na relação com o doente/utente; 8-o doente procure uma relação íntima com o estudante e 9-sobrecarga, os mesmos que resultaram da adaptação para a língua portuguesa do questionário KEZKAK realizada em 2008 por Barroso e colaboradores. De acordo com estes autores, o fator 1 "falta de competência” está relacionado com a perceção do estudante sobre a sua competência para executar com qualidade os cuidados de enfermagem. Verificou-se ser o fator mais stressante e integra os itens 15,16 , $13,4,2,6,26,1,3,17$ e 5 , relacionados com o receio de errar, não ser capaz de ajudar o doente/utente e de o prejudicar ou causar dano. Pode ser um fator que gradualmente diminua a intensidade stressante ao longo do curso à medida que os estudantes adquiram competências e experiência. Quanto ao fator 2, "contacto com o sofrimento", diz respeito à forma como os estudantes percecionam a morte e sofrimento, integrando os itens: 27, 39, 18, 10, 9, 31, 32, 29, 14, 34, que se referem a ver morrer um doente ou estar com um doente terminal e família comunicar uma má noticia ou ter que realizar procedimentos que causam dor ao doente. Em virtude das situações de contacto com a dor e sofrimento serem intrínsecas ao cuidar na profissão de enfermagem, e como tal persistentes ao longo da toda a vida profissional, os autores consideram importante que durante a formação inicial se promovam o desenvolvimento de competências e estratégias que permitam uma melhor adaptação face às situações de dor, morte e sofrimento.
Por outro lado, o feedback dos orientadores, a orientar para o alcance dos objetivos e para a mobilização de estratégias de coping, pode ajudar a reduzir o stress e a melhorar o desempenho do estudante. $\mathrm{O}$ fator 4, "impotência e incerteza", que integra os itens: $23,38,14,41,36,6,32,3,17,2,20$, que dizem respeito a situações como a de não encontrar o médico quando é necessária a sua presença, relaciona-se com o sentimento de ajudar as pessoas e ter soluções para todas as situações com que se deparam, receber ordens contraditórias e a diferença entre o que aprendem na escola e o que fazem na prática. Contudo, nos cuidados de enfermagem, os estudantes deparam-se com situações diante das quais se sentem impotentes e com muitas incerteza quanto à evolução das situações clínicas e eficácia dos tratamentos. Este fator permitiu sublinhar a preocupação dos estudantes quanto às diferenças entre o que é aprendido na escola e a realidade da prática dos cuidados. No tocante ao fator 5, "não controlar a relação como o doente/ utente", dizem respeito os itens: 5, 33, 7, 30, 29, 39, 17, 20, que é constituído por situações como não saber o que dizer e como terminar a conversa com o doente $\mathrm{e}$ estar diante de uma situação de urgência. A este fator está subjacente a comunicação com o doente/utente, levando os autores a sugerir uma maior acuidade para o desenvolvimento de capacidades relacionais e comunicacionais. Relativamente ao fator 6 , "implicação emocional", traduzido para a língua portuguesa por "envolvimento emocional" é composto pelos itens: 21, $8,22,31$, referem-se a situações em que as emoções do doente se projetam no estudante, estar com um doente e ter de ocultar uma má notícia e o forte envolvimento emocional durante a prestação de cuidados. Com efeito, a falta de preparação dos estudantes para comunicar e expressar sentimentos e o estilo predominante de comunicação em enfermagem, centrado na tarefa e impessoal, pode gerar atitudes defensivas (medo de vitimização) que comportem a relação terapêutica. Neste contexto, é importante desenvolver capacidades que permitam o estabelecimento de uma "distância segura" e competências relacionais e comunicacionais que promovam uma efetiva relação de ajuda. $O$ fator 7 "prejudicar-se na relação com o doente/utente", traduzido para a língua portuguesa por "ser magoado na relação com o doente/utente" ao integrar os itens: $11,24,26,15,14$, referem-se a situações como o receio dos estudantes serem maltratados pelo doente, a falta de respeito e picarem-se com uma agulha infetada. 
As consequências negativas que podem advir da relação com o doente constituem fonte de stress e, como tal, interferir na aprendizagem e desenvolvimento pessoal dos estudantes. No que se refere ao fator 8 , "o doente procura uma relação íntima com o estudante", traduzido para a língua portuguesa por "ser magoado na relação com o doente" compreende apenas dois itens: 40 E 37 referem-se a situações em que o doente toca certas partes do corpo do estudante e que o doente de outro sexo se insinue ao estudante.

Apontam para aspetos do comportamento que $o$ doente/utente tem no sentido de procurar uma relação mais próxima e íntima com o estudante. De facto, inerente às profissões da saúde e de modo mais particular à profissão de enfermagem, está a atitude profissional de aceitação incondicional e o desejo de reconhecimento (ser aceite) pelo doente, preocupações que sendo extensíveis aos estudantes exigem dos profissionais o acompanhamento no desenvolvimento dos instrumentos básicos de comunicação e na adoção de estratégias de coping para ultrapassar estas situações. Por último, o fator 9 "sobrecarga" integra os itens: 35, $36,34,30,31$, refere-se a situações de excesso de trabalho durante um turno, receber ordens contraditórias, trabalhar com doentes agressivos, prestar cuidados a um doente com o qual é difícil comunicar e estar na presença de doentes aos quais que se ocultou uma má notícia. Por último aplicou-se o Inventário de Burnout de Maslach (MBI), elaborado por Maslach e Jackson em 1978, foi projetado para avaliar a Síndrome de Burnout em trabalhadores.

A sua construção partiu de duas dimensões, exaustão emocional e despersonalização, sendo que a terceira dimensão, realização profissional, surgiu após um estudo desenvolvido com centenas de pessoas de uma ampla gama de profissionais (Maslach, 1993) (Carlotto \& Câmara, 2007). Inicialmente, o inventário possuía 47 itens que foram administrados numa amostra de 605 sujeitos de várias ocupações profissionais. Dez fatores emergiram e, por meio de uma avaliação criteriosa, foram eliminados seis deles, juntamente com 24 itens que não possuíam peso fatorial superior a 0,40. Após a aplicação numa nova amostra de 420 sujeitos com perfil igual ao anterior, os mesmos quatro fatores emergiram, mas penas três destes apresentaram significância empírica (Maslach \& Jackson, 1981, cit. por Carlotto \& Câmara, 2007). A consistência interna das três dimensões do inventário é satisfatória, pois apresenta um alfa de Cronbach que vai desde 0,71 até 0,90 e os coeficientes de teste e reteste vão de 0,60 a 0,80 em períodos de até um mês (Maslach \& Jackson, 1981, cit. por Carlotto \& Câmara, 2007).
O MBI avalia como o trabalhador vivencia o seu trabalho, de acordo com três dimensões conceituais: exaustão emocional, realização profissional e despersonalização. Na sua primeira versão, o inventário avaliava a intensidade e a frequência das respostas com uma escala de pontuação do tipo Likert, variando de 0 a 6 (Maslach \& Jackson, 1981; Maslach \& Leiter, 1997). A segunda edição do MBI, realizada em 1986, passou a utilizar apenas a avaliação da frequência, pois foi detetada a existência de alta associação entre as duas escalas, sendo que muitos estudos apontaram uma correlação superior a 0,80 (Maslach \& Jackson, 1986; Maslach, 1993; Moreno e cols.,1997, cit. por Carlotto \& Câmara, 2007). O MBI é um instrumento utilizado exclusivamente para a avaliação da síndrome, não tendo em consideração os elementos antecedentes e as consequências do seu processo. Avalia índices de Burnout de acordo com os scores de cada dimensão, sendo que altos scores em exaustão emocional e despersonalização e baixos scores em realização profissional (esta subescala é inversa) indicam alto nível de Burnout (Maslach \& Jackson, 1986). Gil-Monte e Peiró (2008) reforçam a importância de avaliar o MBI como um constructo tridimensional, ou seja, as três dimensões devem ser avaliadas e consideradas, a fim de manter sua perspetiva de síndrome. A escala utlizada neste trabalho é constituída por 22 itens, subdivididos por três fatores: exaustão emocional que é constituída pelos itens 1, 2, 3, 4, 5, 6 e 7; a despersonalização contém os itens $8,9,10,11,12,13$ e 14 e o fator 3 realização pessoal pelos itens $15,16,17,18,19$, 20,21 e 22 .

\section{RESULTADOS}

Os resultados permitiram traçar um perfil sociodemográfico da amostra dos 236 estudantes de enfermagem em ensino clínico, sendo a maioria do sexo feminino (78.4\%) com uma idade média 21.17 anos ( \pm .2 .487 anos), verificando-se que os estudantes do sexo masculino são em média $(M=21.56$ anos $\pm .2 .574$ anos), mais velhos do que as do sexo feminino. Prevalecem os estudantes solteiros (96.6\%), os residentes em zona urbana $(65.3 \%)$ e os que coabitam com os amigos/residência (54.2\%) em tempo de aulas, seguidos dos que vivem com a família (40.7\%).

Os resultados mais significativos obtidos em relação ao stress nos estudantes de enfermagem em ensino clínico revelam, para o total do stress, um mínimo de 12.00 e um máximo de 99.00 , ao que corresponde uma média de $56.58( \pm 16.576)$. 
Fazendo-se uma leitura global dos resultados, o valor médio mais elevado é registado na dimensão "Não controlar a relação com o doente/utente" 49.36 ( \pm 16.458$)$, onde o mínimo é 8.33 e o máximo 75.00, o que revela que esta dimensão é um fator de stress para os estudantes. Segue-se, em termos de média, a dimensão "Falta de competência" ( $M=48.55 ; \pm 21.043)$, registando-se um mínimo de 0.00 e um máximo de 75.00. Os coeficientes de variação indiciam uma dispersão elevada face às médias encontradas. Os resultados obtidos em relação ao Burnout nos estudantes de enfermagem em ensino clínico revelam um mínimo de 0.00 e um máximo de 86.67 para a exaustão emocional, ao que corresponde uma média de 25.48 $( \pm 16.222)$. Na despersonalização o mínimo encontrado é 0.00 e o máximo 83.33 ( \pm 16.222$)$, com uma média de $18.82(\mathrm{Dp} .=15.124)$. Já na realização profissional, o mínimo registado é 31.25 e o máximo 100.00, com uma média de 65.04 ( \pm 14.033$)$.

Os coeficientes de variação indiciam uma dispersão elevada, para a exaustão emocional e despersonalização, e uma dispersão baixa, face às médias encontradas. Em relação aos resultados resultantes da análise inferencial e respondendo às questões iniciais, De que modo as variáveis sociodemográficas interferem no Burnout nos estudantes de enfermagem em ensino clínico? Pela aplicação do teste de U-Mann-Whitney, pode considerar-se que a relação entre as variáveis é estatisticamente significativa na despersonalização, existindo relação entre o sexo e a despersonalização $(\mathrm{p}=0.008)$. Que variáveis académicas interferem no Burnout nos estudantes de enfermagem em ensino clínico? Existe relação estatística entre a realização profissional e a coabitação, conforme se observou pela aplicação do teste de Kruskal-Wallis (X2=6.996; $\mathrm{p}=0.030)$.

\section{DISCUSSÃO}

Apurou-se uma prevalência de baixo Burnout nos estudantes, com percentuais mais elevados em todas as variáveis sociodemográficas e académicas. Estes resultados poderão ser explicados com o facto de não se terem verificado um predomínio de stress moderado (47.0\%) na amostra em estudo. Neste âmbito, salientase que as causas do Burnout são multifatoriais, conforme refere Benavides-Pereira (2002). Trata-se da confluência de características pessoais, do tipo de atividade realizada e da constelação de variáveis oriundas da instituição onde o trabalho é realizado.
Os mesmos autores referem que elevados níveis de stress resultam em Burnout, o que não parece ser uma situação aplicada à maioria os estudantes de enfermagem do presente estudo. Alguns estudos confirmam que os estudantes de enfermagem em ensino clínico são confrontados com uma grande variedade de situações vulneráveis, experienciando níveis de stress e ansiedade com maior intensidade (Magalhães, 2004; Barroso, 2009), com repercussões negativas sobre o seu desempenho, saúde física e bem-estar emocional, sintomas depressivos e até Burnout, estando-lhe associado o medo de fracassar.

Há a salvaguardar que, segundo Pocinho e Garcia (2008), o stress ocupacional ocorre quando existe a perceção, por parte da pessoa, da sua incapacidade para realizar as tarefas solicitadas, resultando em sofrimento, mal-estar e num sentimento de incapacidade para enfrentar as situações de trabalho. Todavia, salvaguarda-se que, no presente estudo, os resultados sugerem um predomínio de stress moderado, sendo menor o percentual de casos stress elevado, o que pode justificar a pouca prevalência de Burnout. Na mesma linha, Freitas (2012) observou que apenas 20,07\% dos estudantes apresentam sinais de Burnout. No que se refere à segunda questão de investigação, através da qual se procurava saber de que modo as variáveis sociodemográficas interferem no Burnout nos estudantes de enfermagem em ensino clínico, começa-se por referir os estudantes do sexo masculino os que revelaram valores de ordenação média mais elevados em todas as dimensões do Burnout, sobretudo na despersonalização $(\mathrm{OM}=140.92)$, seguindo-se a exaustão emocional $(\mathrm{OM}=132.56)$. As estudantes apresentaram mais realização profissional $(\mathrm{OM}=118.07)$. Inferiu-se que existe uma relação estatisticamente significativa entre o sexo e a despersonalização $(\mathrm{p}=0.008)$. Freitas (2012), no seu estudo, também verificou que os estudantes do sexo masculino são os que revelaram mais sintomas de exaustão emocional e despersonalização, resultantes do desgaste vivenciado em algumas situações quotidianas dos ensinos clínicos. Contrariamente, Custódio, Pereira e Seco (2009) verificaram que as raparigas evidenciam níveis sintomas de exaustão emocional comparativamente aos rapazes, sendo estas, inclusive, a revelar índices mais elevados de stress. Constatou-se, de igual modo, que os estudantes na faixa etária dos 22-24 anos são os que revelam níveis mais elevados de exaustão emocional $(\mathrm{OM}=122.92)$ e de despersonalização (OM=122.09), sendo os estudantes mais velhos a revelarem um nível de realização profissional mais elevado $(\mathrm{OM}=132.83)$. 
Verificou-se que existe relação estatística entre a exaustão emocional e a idade (X2=9.916; $\mathrm{p}=0.007)$. Estes resultados poderão ser explicados com o facto de os estudantes mais velhos já possuírem mais estrutura emocional, advinda de maior maturidade inclusive, em termos de experiência de ensinos clínicos, do que os estudantes mais novos. Barroso (2009) refere, a este propósito, que à medida que aumenta a idade os estudantes vão, nos seus processos desenvolvimentais, adquirindo maturidade e maior familiaridade com as situações, orientadores e colegas e, consequentemente, sentem-se menos "ameaçados", estando na posse de mais estratégias de coping, que lhes permitem enfrentar melhor as situações mais desgastante, sem que possam entrar em exaustão emocional.

Todavia, Garro, Camillo e Nóbrega (2006), através do seu estudo, verificaram que os estudantes mais velhos, no $4^{\circ}$ ano, são os que se sentem mais despreparados face à saturação do mercado de trabalho, bem como por já serem praticamente profissionais sentem medo de atuarem sozinhos sem apoio dos orientadores. Concluíram que os estudantes dos $10^{\circ}$ e $3 .^{\circ}$ anos apresentam menos sintomas indicativos de Burnout. Em relação à terceira questão de investigação, através da qual se procurou saber que variáveis académicas interferem no Burnout nos estudantes de enfermagem em ensino clínico, apurou-se que apenas a coabitação teve interferência estatística, sendo os estudantes que vivem sozinhos em tempo de aulas os que demonstraram níveis mais elevados de exaustão emocional $(\mathrm{OM}=130.17)$ e, sobretudo, de despersonalização (OM=160.38). Constata-se que os estudantes a coabitarem com a família são os que apresentam mais realização profissional $(\mathrm{OM}=128.28)$. Deste modo, os dados empíricos indicaram que existe relação estatística entre a realização profissional e a coabitação (X2=6.996; $\mathrm{p}=0.030)$. Estes resultados poderão ser justificados com o facto de os estudantes que vivem sozinhos não têm tanto suporte social, como o familiar e o apoio dos amigos/colegas. Um estudo conduzido por Costa e Leal (2006), os estudantes que saíram do ambiente familiar e passaram a viver sozinhos, após a entrada no ensino superior, tinham tendência para sentirem mais ansiedade e apresentarem mais problemas de isolamento/solidão e irritabilidade, contrariamente aos que continuaram a viver com a família, que mostravam uma melhor adaptação académica em geral, eram mais otimistas, apresentavam uma maior estabilidade afetiva, melhor equilíbrio emocional, o que pode justificar os resultados encontrados no presente estudo.

\section{CONCLUSÃO}

Dos resultados do nosso estudo, podemos afirmar que os estudantes de enfermagem revelam que há situações indutoras de stress em contexto de ensino clínico. No entanto, com base nos resultados apurados verificouse um predomínio de stress moderado, onde sobressaem os estudantes do $4^{\circ}$ ano e os do $3^{\circ}$ ano. Apesar de se ter constatado que há um predomínio de stress moderado, apurou-se que uma grande parte dos estudantes revelou stress intenso, sobretudo os que se encontram nos $3^{\circ}$ e $4^{\circ}$ anos. Outra situação a referenciar é a de que alguns dos estudantes em ensino clínico de Pediatria/Obstetrícia foram os que mais revelam stress intenso perante as situações vivenciadas, secundados pelos que estão no ensino clínico de Médico-cirúrgica 4/Comunitária 3. Neste âmbito faz-se alusão ao estudo de Barroso (2009) onde ficou demonstrado também que alguns estudantes do $4 .^{\circ}$ ano, pela proximidade com o mundo do trabalho, apresentam níveis elevados de stress. Pacheco (2008) salienta que um stressor corresponde a qualquer situação provocadora de um estado emocional forte que conduza a uma quebra do equilíbrio interno e externo, que exija do estudante um ajustamento ao ambiente. Assim, o mesmo autor refere algumas as situações de stress experienciadas nos ensinos clínicos, as quais foram corroboradas no presente estudo, designadamente: desconhecimento da estrutura e orgânica do serviço, relação entre colegas/equipa, avaliação, relação entre o estudante com o orientador e com o professor, as quais resultam em alterações fisiológicas, emocionais e comportamentais, favorecedoras de uma diminuição da saúde e do bemestar dos estudantes.

Averiguou-se que o valor médio mais elevado, em termos de situações vivenciadas em ensino clínico e que possam causar stress aos estudantes, foi "Não controlar a relação com o doente/utente", seguindose a "Falta de competência" $(M=48.55 ; \pm 21.043)$ e a "Relação com o supervisor e colegas", "Sobrecarga" e a "Impotência e a incerteza", o que foi corroborado pelos discursos de alguns estudantes. Neste sentido, Rocha e Carvalho (2013) salientam que a formação recebida durante a aprendizagem profissional possibilita a estruturação da identidade profissional e esta é resultante de um subsequente processo de retradução e de reajustamento, no confronto com as experiências concretas nos contextos de ensino clínico. Pode dizerse que há conformidade entre as respostas obtidas, pois as situações geradoras de stress relacionam-se com o ambiente clínico e com a supervisão clínica. 
No que se refere ao ambiente clínico, sobressaiu a relação com o doente, o que lhes pode gerar uma grande tensão emocional. Sobressaiu também a segurança dos cuidados, tendo sido mais valorizado os erros com a medicação, na medida em que o erro na administração da medicação pode por em risco a vida do doente, bem como a impotência e a incerteza face a determinadas situações. Esses fatores geradores de stress foram identificados responsáveis por sobrecarga física e psicológicas, com interferência no bem-estar emocional dos estudantes. Em síntese, ficou demonstrado que existem princípios que se aplicam à realidade do ensino clínico de enfermagem, destacando-se o princípio da relação interpessoal e o princípio da relação intercontextual, os quais podem resultar em elevados níveis de stress, caso os estudantes de enfermagem não tenham acesso a estratégias de coping que os façam superar esses fatores, dando lugar à denominada "adaptação", pois, caso contrário, se os estudantes não os conseguirem superar podem mesmo atingir o Burnout.

\section{REFERÊNCIAS BIBLIOGRÁFICAS}

Barroso, I. M. A. C. (2009). O ensino clínico no curso de licenciatura em enfermagem: Estudo sobre as experiências de aprendizagem, situações e fatores geradores de stresse nos estudantes (Dissertação de mestrado, Universidade do Porto). Acedido em https:// repositorio-aberto.up.pt/bitstream/10216/20159/2/ DissertaoIsabelBarroso.pdf

Benevides-Pereira, A. M. T. (2002). O processo de adoecer pelo trabalho. In A. M. T. Benevides-Pereira (Org.), Burnout: Quando o trabalho ameaça o bemestar do trabalhador. São Paulo: Casa do Psicólogo.

Carloto, M. S. \& Câmara, S. G. (2007). Preditores da síndrome de Burnout em professores. Revista Psicologia Escolar e Educacional, 11(1), 101-110.

Costa, E. S., \& Leal, I. P. (2006). Estratégias de coping em estudantes do ensino superior. Análise Psicológica, 24(2), 189-199.

Custódio, S., Pereira, A., \& Seco, G. (2009). Stress e estratégias de coping dos estudantes de enfermagem em ensino clínico. In Actas do X Congresso Internacional Galego-Português de Psicopedagogia (pp. 4670-4683).
Ferreira, F. C. V. P. (2015). A aprendizagem decorrente de vidas havidas e vidas a haver. Porto: Porto Editora.

Freitas, E. O. (2012). Estresse, coping, Burnout, sintomas depressivos e hardiness entre discentes de enfermagem. (Dissertação de mestrado, Universidade Federal de Santa Maria). Acedido em: http:// cascavel.ufsm.br/tede/tde_arquivos/33/TDE-201210-18T112449Z-3762/Publico/FREITAS, \% 20 ETIANE\%20DE\%20OLIVEIRA.pdf

Garro, I. M. B., Camillo, S. O., \& Nobrega, M. P. S. S. (2006). Depressão em graduandos de enfermagem. Acta Paulista de Enfermagem, 19(2), 162-167. Acedido em http://www.scielo.br/pdf/ape/v19n2/a07v19n2.pdf

Gil-Monte, P. R. (2008). El síndrome de quemarse por el trabajo, burnout, como fenómeno transcultural. Informació Psicológica, 91/92, 4-11.

Magalhães, C. M. S. (2004). Stresse dos alunos de enfermagem em ensino clínico: Estudo exploratório descritivo (Dissertação de mestrado, Universidade do Porto). Acedido em https://repositorio-aberto.up.pt/ bitstream/10216/9627/3/5013_TM_01_P.pdf

Maslach, C. (1993). Burnout: A multidimensional perspective. In W. B. Schaufeli, C. Maslach \& T. Marek (eds.), Professional burnout: Recent developments in theory and research (pp. 19-32). London: Taylor \& Francis.

Pacheco, A. C. L. (2008). Ansiedade, depressão, stress, estratégias de coping e suporte social em enfermeiros que trabalham por turnos e em horário fixo diurno (Dissertação de mestrado, Universidade Lusófona de Humanidades e Tecnologias). Acedido em http://recil.grupolusofona.pt/bitstream/handle/10437/4996/ ANACAROLINACOELHOLEITEPACHECO 1. pdf? sequence $=1$

Pocinho, M. D., \& Garcia, J. C. (2008). Impacto psicosocial de la tecnologia de información y comunicación TIC: Tecnoestrés, danos físicos y satisfacción laboral. Acta Colombiana de Psicologia, 11(2), 127-139.

Rocha, I. A. R. S., \& Carvalho, A. L. R. F. (2013). Reflexões sobre relação e interação supervisiva em ensino clínico de enfermagem. RevRene: Revista da Rede de Enfermagem do Nordeste, 14(2), 428-37. Acedido em http://www.redalyc.org/html/3240/324027986019/index.html 
Serra, M. N. (2008). Aprender a ser enfermeiro: Identidade profissional em estudantes de enfermagem. Revista de Ciências de Educação, 5, 69-80.
Zupiria Gorostidi, X., Uranga Iturriotz, M. J., Alberdi Erize, M. J., \& Barandiaran Lasa, M. (2003). KEZKAK: Cuestionario bilingüe de estresores de los estudiantes de enfermería en las prácticas clínicas. Gaceta Sanitaria, 17(1), 37-51.

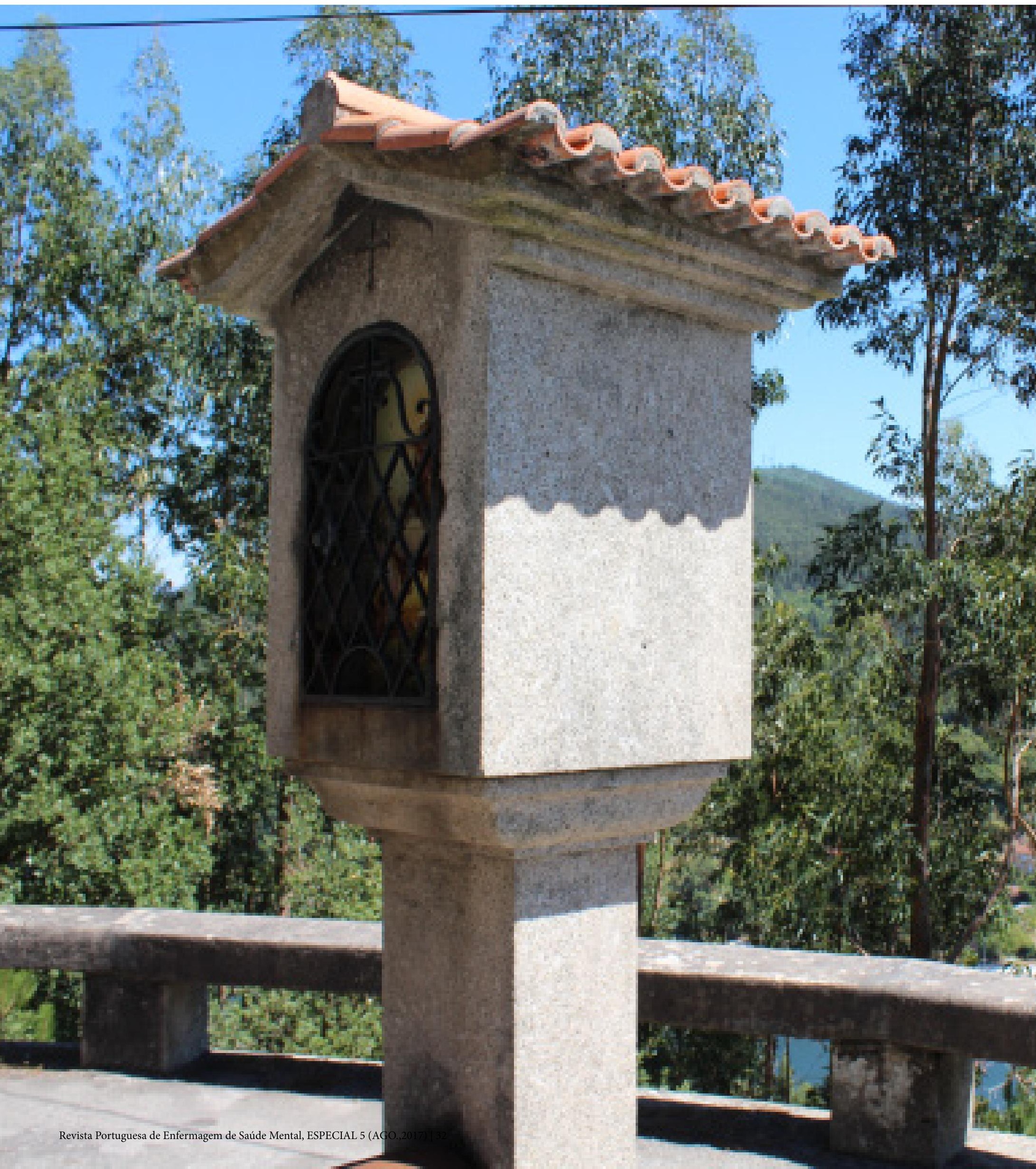

